\title{
Refining CRISPR-based genome and epigenome editing off-targets
}

\author{
Yonglun Luo
}

Received: 28 May 2019 / Accepted: 12 June 2019 / Published online: 22 June 2019

(C) Springer Nature B.V. 2019

To date, it has been broadly demonstrated and well proven that the CRISPR-derived genome and epigenome editing technologies have completely revolutionised and significantly accelerated discoveries and breakthroughs in gene therapy, animal modelling, drug screening, functional genomics etc. Similar to other gene editing tools such as ZFN and TALEN, the CRISPR gene editing tool has two important characteristics: (i) programmable binding to a specific locus in the genome of a living cell/organism; (ii) enzymatic introduction of a double-strand break (DSB) within or adjacent to the binding site. The beauty of CRISPR gene editing system is that it contains a programmable small RNA, known as the guide RNA (gRNA), which directs a sole CRISPR-associated protein (Cas) to the target site where it introduces a DSB. In mammalian cells, unrepaired DSBs are detrimental, subsequently leading

Y. Luo

BGI-Shenzhen, Shenzhen 518083, China

Y. Luo

Lars Bolund Institute of Regenerative Medicine, BGI-Qingdao, Qingdao 266555, China

Y. Luo

Department of Biomedicine, Aarhus University, 8000 Aarhus, Denmark

Y. Luo $(\bowtie)$

China National GeneBank, BGI-Shenzhen, Shenzhen 518120,

China

e-mail: luoyonglun@genomics.cn

e-mail: alun@biomed.au.dk to genomic instability and chromosomal aberrations. Cells have thus developed self-protecting and systematic DNA damage repair mechanisms to repair the DSBs. In mammalian cells, DSBs are mainly repaired by the non-homologous end joining (NHEJ) pathway leading to introduction of small deletion or insertions (indels) at the break site after repairing. The introduction of DSBs also stimulates the homology-directed repair (HDR) machinery in mammalian cells. However, compared with the NHEJ pathway, the efficiency of DSB repair by HDR is approximately 40 folds lower (Weinstock and Jasin 2006). The principle of all current gene editing technologies is based on hijacking programmable DNA endonucleases to introduce specific DSBs and activate the cellular DSB repair machinery. While CRISPR technology has been so broadly used, its specificity versus risk of off-target effects is consistently ranked as the top concern especially in clinically orientated applications.

In this editorial, I would like to focus the CRISPR/ Cas 9 off-targets into two main categories: (1) Off-target cleavage and (2) Off-target modification.

The first problem, off-target cleavage, refers to the introduction of either single-strand nickings by Cas9 nickase or DSBs by wild-type Cas9 at unintended genomic loci. This is the typical category of off-targets that most applications are addressing. The Cas9 protein contains two catalytic domains, RuvC and $\mathrm{HNH}$, which are responsible for cleaving the non-complementary and complementary strand of the target DNA, respectively (Nishimasu et al. 2014). Introducing catalytically inactivating mutations to one or both domains has generated Cas9 nickase (nCas9) or dead Cas9 (dCas9). The 
CRISPR/Cas9 system has evolved as a quite stringent and high-fidelity system to ensure specificity. Dependence on a PAM-sequence and R-loop formation for activation of the Cas9 nuclease activity is crucial. However, the propensity for Cas9 to tolerate certain mismatches (up to $3 \mathrm{bp}$ for SpCas9), between the gRNA spacer and the target site, generates the potential of introducing DSBs to similar loci across the genome (Cong et al. 2013). It was demonstrated experimentally that up to a few hundred off-target cleavages could be introduced by a single CRISPR gRNA (Tsai et al. 2015). Thus, carefully selecting a gRNA of high specificity is necessary and many CRISPR gRNA designing softwares have been developed for picking the best gRNA of high specificity and activity (Heidenreich and Zhang 2016). Apart from the number of mismatches, other challenges appear such as the position and combination of mismatches, the secondary structure of the gRNA spacer and the chromatin accessibility at the target site, which all affect CRISPR/Cas9 cleavage activity (Jensen et al. 2017). Together, these challenges make the prediction of CRISPR activity and off-target effects far more complicated - and it is thus not surprising to discover a discordance between in silico predicted and experimentally validated off-targets (Tsai et al. 2015). Many approaches have been developed to reduce the CRISPR off-target cleavage effect. For example, truncation of the CRISPR gRNA spacer length to 18-19 nt (Fu et al. 2014), Cas9 proteins with weakened DNA binding activity (e.g. eSpCas9, SpCas9-HF1, xSpCas9) and self-restriction of the CRISPR systems to shorten the time of Cas 9 expression (Chen et al. 2016) have been tried with some success. Another important development in our understanding of the CRISPR off-target cleavage effect are methods for detecting the off-target cleavage in a genome-wide manner such as GUIDE-seq, Digenome-Seq and CIRCLE-seq (Kim et al. 2015; Tsai et al. 2017; Tsai et al. 2015).

The second problem is off-target modification. One of the most important characteristics of the Cas 9 protein is the flexibility it provides for engineering. Fusion of effector proteins to either the $\mathrm{N}$-terminus or $\mathrm{C}$-terminus of Cas9, nCas9 or dCas9 has greatly expanded the applications of CRISPR-Cas9 in genetic manipulation. For instance, CRISPR/dCas9 has been successfully engineered for activation (CRISPRa) or inhibition (CRISPRi) of endogenous gene expression by fusing dCas9 to transcriptional activator or repressor domains, respectively (Qi et al. 2013; Xiong et al. 2017). To achieve epigenome editing, the CRISPR/dCas9 system has even been successfully engineered for RNA-guided histone acetylation (Hilton et al. 2015), DNA methylation (CRISPRme) (Lin et al. 2018) and DNA demethylation (Xu et al. 2016). RNA-guided base editing, achieved by fusing either dCas9 or nCas 9 to deaminases, has become another broadly explored application over the last few years. Compared with non-targeted DNA cleavage, the non-targeted DNA binding is more abundant and broadly distributed. This is mainly due to the fact that the Cas9 protein has greater tolerance to mismatches and has a smaller seed region $(5 \mathrm{nt})$ for DNA binding (Tsai et al. 2015). Thus, a significant number of the aforementioned CRISPR-based applications, which rely on RNA-guided DNA binding, undoubtedly come with more potential off-target modifications to histone/DNA/RNA. For CRISPRa or CRISPRi, the dCas9 effectors must bind to or near the promoter/enhancer regions, the off-target effects might be less prominent and sometimes difficult to capture as most of those off-target binding will not affect gene expression. However, for histone modifications or DNA methylations, the dCas9-based epigenetic editors will leave some footprint in the genome, altering either the histone acetylation or the DNA methylation pattern. Previously, we found that expression of dCas9DNMT3A-CD or dCas9-DNMT3B-CD can cause substantial unspecific methylations across the whole genome (Lin et al. 2018). A large proportion of these unspecific methylated sites are located in open chromatin regions (euchromatin), promoters and 5' untranslated regions of actively expressed genes. More importantly, many of these unspecific methylations are independent of gRNAs. For CRISPR-mediated base editing, e.g. $\mathrm{BE} 3$ and BE4 CBEs, several studies have recently reported transcriptome-wide off-target RNA editing (Grunewald et al. 2019; Zuo et al. 2019). However, it is not too surprising to discover these genome-wide offtarget effects caused by the CRISPR-based epigenetic/ genetic editors. Almost all these CRISPR-derived epigenetic editing tools are based on hijacking the RNAguided DNA binding feature of dCas9/nCas 9 to bring the effector (enzymes or catalytic domains) to the target site. It should be noted that most of these effectors are constantly active regardless of their specific binding to DNA or not. Thus, off-target activity of these CRISPRderived effectors could simply be caused by stochastic interaction between the dCas9 fusion effectors and the endogenous DNA. 
However, while it is essential to be aware of all these potential off-target effects caused by CRISPR-derived genetic/epigenetic editors, this should not prevent use of the systems but lead to caution. Great efforts have been taken to reduce the off-target modifications. The most frequently adapted approach is through structure-guided neutralisation of positively charged residues (e.g. Lysine (K), Arginine (R)) to nonpolar amino acids (e.g. Alanine (A)) in both the dCas 9 protein and the effector domains, e.g. eSpCas9, BE3-R33A, BE3-R33A/K34A (Grunewald et al. 2019; Slaymaker et al. 2016). These engineered variants greatly reduce the DNA binding affinity of the dCas9-fusion effectors, making the dCas9 fusion effector proteins more dependent on RNAguided DNA binding. Another approach, demonstrated as beneficial for reducing the off-target modification effects, is based on controlling the temporal and spatial expression of the effector proteins with inducible systems (Chen et al. 2017). As the whole CRISPR genome and epigenome editing technology field is developing dramatically, novel CRISPR-based tools, improvements to CRISPR specificity and even new generation of gene editing tools are expected in the near future.

Acknowledgements Great thanks to Fred Dubee and Lars Bolund for their valuable comments and discussions.

Funding information CRISPR gene editing projects at the Lars Bolund Institute of Regenerative Medicine (LBI) are partially supported by the Guangdong Provincial Key Laboratory of Genome Read and Write (No. 2017B030301011).

\section{References}

Chen TJ, Gao D, Zhang RS, Zeng GH, Yan H, Lim EJ, et al. Chemically controlled epigenome editing through an inducible dCas9 system. J Am Chem Soc. 2017;139:11337-40.

Chen Y, Liu X, Zhang Y, Wang H, Ying H, Liu M, et al. A selfrestricted CRISPR system to reduce off-target effects. Mol Ther. 2016;24:1508-10.

Cong L, Ran FA, Cox D, Lin S, Barretto R, Habib N, et al. Multiplex genome engineering using CRISPR/Cas systems. Science. 2013;339:819-23.

Fu Y, Sander JD, Reyon D, Cascio VM, Joung JK. Improving CRISPR-Cas nuclease specificity using truncated guide RNAs. Nat Biotechnol. 2014;32:279-84.
Grunewald J, Zhou R, Garcia SP, Iyer S, Lareau CA, Aryee MJ, et al. Transcriptome-wide off-target RNA editing induced by CRISPR-guided DNA base editors. Nature. 2019;569:433-7.

Heidenreich M, Zhang F. Applications of CRISPR-Cas systems in neuroscience. Nat Rev Neurosci. 2016;17:36-44.

Hilton IB, D'Ippolito AM, Vockley CM, Thakore PI, Crawford GE, Reddy TE, et al. Epigenome editing by a CRISPR-Cas9based acetyltransferase activates genes from promoters and enhancers. Nat Biotechnol. 2015;33:510-7.

Jensen KT, Floe L, Petersen TS, Huang J, Xu F, Bolund L, et al. Chromatin accessibility and guide sequence secondary structure affect CRISPR-Cas9 gene editing efficiency. FEBS Lett. 2017;591:1892-901.

Kim D, Bae S, Park J, Kim E, Kim S, Yu HR, et al. Digenome-seq: genome-wide profiling of CRISPR-Cas9 off-target effects in human cells. Nat Methods. 2015;12:237-43 231 p following 243.

Lin L, Liu Y, Xu F, Huang J, Daugaard TF, Petersen TS, et al. Genome-wide determination of on-target and off-target characteristics for RNA-guided DNA methylation by dCas9 methyltransferases. Gigascience. 2018;7:1-19.

Nishimasu H, Ran FA, Hsu PD, Konermann S, Shehata SI, Dohmae N, et al. Crystal structure of Cas9 in complex with guide RNA and target DNA. Cell. 2014;156:935-49.

Qi LS, Larson MH, Gilbert LA, Doudna JA, Weissman JS, Arkin AP, et al. Repurposing CRISPR as an RNA-guided platform for sequence-specific control of gene expression. Cell. 2013; 152:1173-83.

Slaymaker IM, Gao L, Zetsche B, Scott DA, Yan WX, Zhang F. Rationally engineered Cas 9 nucleases with improved specificity. Science. 2016;351:84-8.

Tsai SQ, Nguyen NT, Malagon-Lopez J, Topkar VV, Aryee MJ, Joung JK. CIRCLE-seq: a highly sensitive in vitro screen for genome-wide CRISPR-Cas9 nuclease off-targets. Nat Methods. 2017;14:607-14.

Tsai SQ, Zheng Z, Nguyen NT, Liebers M, Topkar VV, Thapar V, et al. GUIDE-seq enables genome-wide profiling of offtarget cleavage by CRISPR-Cas nucleases. Nat Biotechnol. 2015;33:187-97.

Weinstock DM, Jasin M. Alternative pathways for the repair of RAG-induced DNA breaks. Mol Cell Biol. 2006;26:131-9.

Xiong K, Zhou Y, Blichfeld KA, Hyttel P, Bolund L, Freude KK, et al. RNA-guided activation of pluripotency genes in human fibroblasts. Cell Rep. 2017;19:189-98.

Xu X, Tao Y, Gao X, Zhang L, Li X, Zou W, et al. A CRISPRbased approach for targeted DNA demethylation. Cell Discov. 2016;2:16009.

Zuo E, Sun Y, Wei W, Yuan T, Ying W, Sun H, et al. Cytosine base editor generates substantial off-target single-nucleotide variants in mouse embryos. Science. 2019;364:289-92.

Publisher's note Springer Nature remains neutral with regard to jurisdictional claims in published maps and institutional affiliations. 